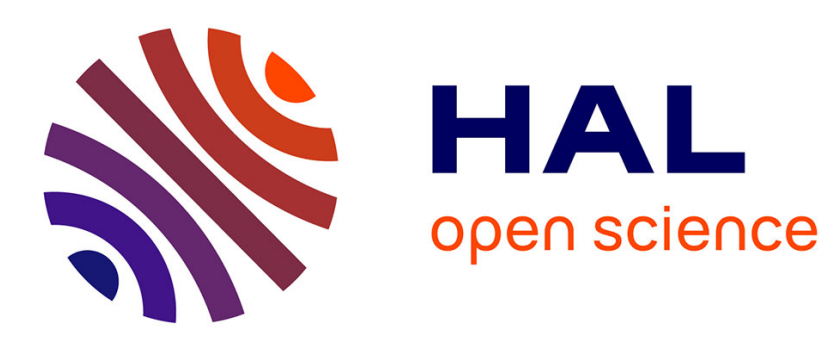

\title{
Stability in the obstacle problem for a shallow shell
}

Alain Léger, Bernadette Miara

\section{To cite this version:}

Alain Léger, Bernadette Miara. Stability in the obstacle problem for a shallow shell. Analysis and Applications, 2016, 14 (2), pp.207-232. 10.1142/S0219530514500225 . hal-01276240

\section{HAL Id: hal-01276240 \\ https://hal.science/hal-01276240}

Submitted on 19 Feb 2016

HAL is a multi-disciplinary open access archive for the deposit and dissemination of scientific research documents, whether they are published or not. The documents may come from teaching and research institutions in France or abroad, or from public or private research centers.
L'archive ouverte pluridisciplinaire HAL, est destinée au dépôt et à la diffusion de documents scientifiques de niveau recherche, publiés ou non, émanant des établissements d'enseignement et de recherche français ou étrangers, des laboratoires publics ou privés. 


\title{
Stability in the obstacle problem for a shallow shell
}

\author{
Alain Léger \\ Laboratoire de Mécanique et d'Acoustique - CNRS \\ 31, Chemin Joseph Aiguier \\ 13402 Marseille Cedex 20, France \\ leger@lma.cnrs-mrs.fr \\ Bernadette Miara \\ Université Paris-Est, Cité Descartes \\ 93160 Noisy-le-Grand Cedex, France \\ bernadette.miara@gmail.com
}

\begin{abstract}
This work deals with the variation of the solution to an obstacle problem with respect to the variation of its parameters. More precisely, a mechanical structure is pushed by some external forces against an obstacle in such a way that the equilibrium solution involves a part of the domain in which the structure is strictly in contact with the obstacle. It is known from the theory of variational inequalities that studying the variation of the solution as the external forces vary amounts to studying the variation of the boundary of this contact zone. This problem has been studied in previous works in the scalar case, and it was open in the general case where the unknown is a vector field, due to the coupling between the components. As a first step, the present work considers the case of a linearly elastic shallow membrane shell where the coupling between the in-plane and normal components of the displacement arises from the curvature.
\end{abstract}

Keywords: Free boundary; obstacle problem; stability; unilateral contact; Nash-Moser theorem.

\section{Introduction}

We are studying a coupled obstacle problem. A mechanical structure is bent over an obstacle by some external distribution of forces so that the natural unknown is made of the three components of the displacement field. The stability notion we are dealing with concerns the variation of the solution when the forces vary, which can be seen as a sensitivity result. In the present case the problem arises from mechanics, but the same kind of problem can be met in other fields of physics such as electromagnetism or electrostatics $[20,2]$. Relatively few works have been done 
in this field. As far as the present writers are aware, they essentially deal with scalar problems in the case of linear operators. In mechanics, the harmonic operator is the simplest model for the out-of-plane component of the displacement of a linearly elastic flat membrane subjected to a distribution of forces normal to its plane in the reference configuration. The stability result for the obstacle problem in the case of the scalar harmonic operator has been given in the pioneer paper by Schaeffer [21]. Years later, the case of the biharmonic operator which, in the scalar case, can be seen as the simplest model for the out-of-plane component of the displacement of a linearly elastic plate at small strains subjected to a distribution of forces normal to its plane, had been solved in [14]. Only one incursion has been done in the field of nonlinear operators in [17] where a quadratic or a cubic term were added to the harmonic operator.

The main qualitative difference with respect to previous studies is that we are now dealing with a problem in which the unknown is a vector field. It is known that in the mechanics of continuous media the three components of the displacement are strongly coupled by the strain tensor, even in the linear case, and that only some very particular geometries may lead to uncoupling. But the obstacle problem in presence of coupling was known as intricate, so that we tried to restrict our attention to the simplest coupled problem we could find: that of a shallow linear membrane shell over a flat obstacle, in which the coupling is only due to the curvature. This means that the present paper could be seen as a first step in the stability analysis of more general coupled problems.

We now outline the main sections of the work.

Section 2 recalls the shell model we are dealing with. We start from two models which have been justified by an asymptotic analysis, on the one hand the nonlinear shallow shell in the bilateral case, and on the other hand the linear shallow shell in the unilateral case. That way we are able to give a linear shell membrane model in unilateral contact with an obstacle. Geometrical assumptions are made: not only the reference configuration is assumed to be shallow, in the sense given in [5], but it is also assumed to be weakly curved, in the sense given in [18]. The latter assumption will be used in the proof of the main result.

Section 3 is then only concerned with the shallow membrane problem. We first give different formulations of the equilibrium problem, when the contribution of the tangential components of the displacement have been replaced by the introduction of an Airy function.

Section 4 is the main part of the paper, it deals with the stability result. We first transform the classical formulation of the equilibrium problem into a problem which connects the boundary of the contact zone with the external force. Then an implicit function argument, which is Nash-Moser Theorem, states that if the external force varies in a set of smooth functions, then the boundary of the contact zone will also vary smoothly in such a way that one have a smooth diffeomorphism between the force and the solution. 


\section{Building the Model}

We start from the Marguerre--von Kármán shell model without obstacle and with classical bilateral boundary conditions which have been justified in [6]. Here the set of equations is simply recalled formally. Additional points concerning the functional framework and the regularity will be given later only in the case of the obstacle problem for the shallow membrane we are going to deal with.

\subsection{The Marguerre-von Kármán shell model}

Let $\Omega$ be a simply connected smooth open set of the plane with smooth boundary $\partial \Omega$ and a $C^{k}$-diffeomorphism $\theta: \bar{\Omega} \rightarrow \mathbb{R}$ which defines the middle surface $S$ of the shell,

$$
S=\left\{\left(x_{1}, x_{2}, \theta\left(x_{1}, x_{2}\right)\right),\left(x_{1}, x_{2}\right) \in \bar{\Omega}\right\} .
$$

The reference configuration of the shell is therefore the domain of $\mathbb{R}^{3}$ defined by

$$
\left\{\left(x_{1}, x_{2}, \theta\left(x_{1}, x_{2}\right)+x_{3} a\left(x_{1}, x_{2}\right)\right),\left(x_{1}, x_{2}\right) \in \bar{\Omega},\left|x_{3}\right| \leq h\right\},
$$

where $a$ is the unit normal to $S$ and $h$ is given as the uniform thickness of the shell, with $h \ll 1$. The displacement of any point of the shell is a vector field $U=\left(u_{1}, u_{2}, u_{3}\right)$, defined by three scalar functions which are the components of the displacement field in the Cartesian framework. However, a more convenient formulation of the equilibrium problem amounts to calculating two scalar functions $u$ and $\varphi$, where $u$ is the vertical component of the displacement (i.e. $u \equiv u_{3}$ ) and $\varphi$ is the Airy function associated with the two in-plane components $u_{1}$ and $u_{2}$, i.e.

$$
\partial_{11} \varphi=N_{22}, \quad \partial_{22} \varphi=N_{11}, \quad \partial_{12} \varphi=-N_{12},
$$

where $N_{\alpha \beta}, \alpha, \beta \in\{1,2\}$, are the components of the resultant of the internal in-plane stresses. Let $f$ be the external applied force, then the equilibria of a Marguerre-von Kármán shell are given by the solution $(u, \varphi)$ of the following problem:

$$
\begin{cases}\frac{2 E h^{3}}{3\left(1-\nu^{2}\right)} \Delta^{2} u-2 h[\varphi, u+\theta]=f & \text { in } \Omega, \\ \Delta^{2} \varphi+\frac{E}{2}[u, u+2 \theta]=0 & \text { in } \Omega, \\ u=\partial_{n} u=0, \quad \varphi=\varphi_{1}, \quad \partial_{n} \varphi=\varphi_{2} & \text { on } \partial \Omega,\end{cases}
$$

where $E$ is the Young modulus, $\nu$ is the Poisson ratio, the index $n$ stands for the unit normal to $\partial \Omega$ directed outwards to $\Omega$, and $[\cdot, \cdot]$ is the Monge-Ampère bracket defined, for any smooth enough functions $g$ and $k$, as

$$
[g, k]=\partial_{11} g \partial_{22} k+\partial_{22} g \partial_{11} k-2 \partial_{12} g \partial_{12} k \text {. }
$$

\subsection{The linearized shallow shell and membrane models}

Let $(\widehat{u}, \widehat{\varphi})$ be a solution to problem $(2.1)$ associated with data $\left(\widehat{f}, \widehat{\varphi}_{1}, \widehat{\varphi}_{2}\right)$, and consider the solution $(\widehat{u}+u, \widehat{\varphi}+\varphi)$ associated with the load $(\widehat{f}+f)$ 
and the same boundary data $\left(\widehat{\varphi}_{1}, \widehat{\varphi}_{2}\right)$. Assume in addition that $\|f\| \ll$ $\|\widehat{f}\|$, and that the solution varies smoothly with respect to the forces. Let us introduce $\widehat{\theta}:=\widehat{u}+\theta$. According to these assumptions, the quadratic terms $[\varphi, u]$ and $[u, u]$ are supposed to be sufficiently small to be neglected, then linearization of the shallow shell problem around $(\widehat{u}, \widehat{\varphi})$ consequently gives:

$$
\begin{cases}\frac{2 E h^{3}}{3\left(1-\nu^{2}\right)} \Delta^{2} u-2 h([\widehat{\varphi}, u]+[\varphi, \widehat{\theta}])=f & \text { in } \Omega, \\ \Delta^{2} \varphi+E[u, \widehat{\theta}]=0 & \text { in } \Omega, \\ u=1, \quad \partial_{\nu} u=0, \quad \varphi=0, \quad \partial_{n} \varphi=0 & \text { on } \partial \Omega .\end{cases}
$$

The linearized shallow membrane problem is then obtained formally by neglecting the bending part in the equations (i.e. neglecting either the term $\Delta^{2} u$, or the contribution of the thinness of the shell since $h^{3} \ll h$ ) and in the associated boundary condition:

$$
\begin{cases}-[\widehat{\varphi}, u]-[\varphi, \widehat{\theta}]=\frac{f}{2 h} & \text { in } \Omega \\ \Delta^{2} \varphi+E[u, \widehat{\theta}]=0 & \text { in } \Omega \\ u=1, \quad \varphi=0, \quad \partial_{n} \varphi=0 & \text { on } \partial \Omega .\end{cases}
$$

Problem (2.3) could be taken either as the membrane problem linearized around $(\widehat{u}, \widehat{\varphi})$, or directly as the equilibrium problem of a linear membrane at small strains.

\subsection{Introduction of unilateral contact conditions}

Introducing unilateral contact conditions in a shallow shell problem has been mathematically justified in $[11,12]$. We start from bilateral problem $(2.3)$. We assume that the shell is above a flat obstacle at the level $x_{3}=0$, and let $\mu$ be a positive measure which represents the reaction of the obstacle onto the shell. This reaction has the mechanical meaning of an external force, which prevents the shell from passing through the obstacle; it is a part of the solution, so that there is no $a$ priori assumption whether it is diffuse or involves an atomic part. We only know that it can be nonzero only at the points of the shell which are in contact with the obstacle, which means that there is no distance interaction, and it is positive which means that the obstacle can only push the points in contact backwards (see $[12])$. Classically, all the points of the shell must be above the obstacle, so that $u(x)$ should be positive everywhere in $\bar{\Omega}$, and it is possible to have $\mu \neq 0$ only when $u(x)=0$. In particular $u>0$ on $\partial \Omega$. We choose for instance without restriction $u=1$ on $\partial \Omega$. These requirements do not depend on whether the structure involves a bending stiffness or not. In all the present work we restrict our attention to the 
membrane case, so that the unilateral problem we are going to deal with reads as follows:

$$
\begin{cases}-[u, \widehat{\varphi}]-[\varphi, \widehat{\theta}]=\frac{f}{2 h}+\mu & \text { in } \Omega, \\ \Delta^{2} \varphi+E[u, \widehat{\theta}]=0 & \text { in } \Omega, \\ \mu \geq 0, \quad u \geq 0, \quad \mu u=0 & \text { in } \Omega \\ u=1, \quad \varphi=0, \quad \partial_{n} \varphi=0 & \text { on } \partial \Omega .\end{cases}
$$

\section{The Obstacle Problem for a Shallow Membrane}

Let $I$ be the subdomain in which the membrane is in contact with the obstacle. In the following, the subdomain $I$ will be referred to as the coincidence set, keeping in mind that $I$ should be written as $I(f)$, and the boundary $\partial I(f)$ of the coincidence set, will be referred to as the free boundary.

It will first be useful to have different formulations of the equilibrium problem. The natural formulation is given in Eq. (2.4), which is exactly equivalent to the following one

$$
\begin{cases}-[u, \widehat{\varphi}]-[\varphi, \widehat{\theta}] \geq \frac{f}{2 h} & \text { in } \Omega, \\ \Delta^{2} \varphi+E[u, \widehat{\theta}]=0 & \text { in } \Omega, \\ u \geq 0, \quad\left(-[u, \widehat{\varphi}]-[\varphi, \widehat{\theta}]-\frac{f}{2 h}\right) u=0 & \text { in } \Omega, \\ u=1, \quad \varphi=0, \quad \partial_{n} \varphi=0 & \text { on } \partial \Omega .\end{cases}
$$

Remark 3.1. Note that the problem of a smooth curved membrane over a flat obstacle is very different from that of a flat membrane over a curved obstacle. As a matter of fact it is easily shown [13] that the latter problem can be changed into the case of a flat membrane over a flat obstacle by changing the distribution of forces and keeping a scalar problem. The vectorial structure in the case of a curved membrane results from the coupling in the strain tensor, due to the curvature of the reference configuration, and cannot be removed by changing the shape of the obstacle or the distribution of forces.

Another formulation could be written using the fact that, due to the definition of the coincidence set $I, \mu$ is equal to zero in $\Omega / I$. This formulation reads formally:

$$
\begin{cases}-[u, \widehat{\varphi}]-[\varphi, \widehat{\theta}]=\frac{f}{2 h} & \text { in } \Omega / I \\ \Delta^{2} \varphi+E[u, \widehat{\theta}]=0 & \text { in } \Omega, \\ u>0 & \text { in } \Omega / I \\ u=0 & \text { in } I, \\ u=1, \quad \varphi=0, \quad \partial_{n} \varphi=0 & \text { on } \partial \Omega\end{cases}
$$


But problem (3.2) is not equivalent to (2.4) or (3.1), since in particular it is easily seen that formulation (3.2) is ill-posed due to some lack of matching conditions at the free boundary $\partial I$.

\subsection{Well-posedness of the obstacle problem for a shallow membrane at small strains}

Let $\psi$ be a $C^{2}(\Omega)$ real function and let us introduce the matrix of second-order derivatives $\left(\begin{array}{ll}+\partial_{22} \psi & -\partial_{12} \psi \\ -\partial_{12} \psi & +\partial_{11} \psi\end{array}\right)$ which we shall denote by $D_{2} \psi$.

We first make the following assumption.

Assumption 3.2. The matrix $D_{2} \widehat{\varphi}$ is positive definite, which is the same as positivity of the Hessian matrix of $\widehat{\varphi}$.

Let us now denote by $a_{\psi}(u, v)$ a real function defined as

$$
a_{\psi}(u, v)=\nabla^{t} u(x)\left(D_{2} \psi(x)\right) \nabla v(x)=\left\langle\nabla^{t} u, \nabla^{t} v\right\rangle_{\psi} .
$$

We introduce the vector space $V(\Omega)=\left\{(v, \psi), v \in H^{1}(\Omega), \psi \in H_{0}^{2}(\Omega)\right\}$ and the closed convex set $K(\Omega)=\{(v, \psi) \in V(\Omega), v=1$ on $\partial \Omega, v \geq 0$ in $\Omega\}$. The variational form of problem (2.4) reads:

$$
\left\{\begin{array}{l}
\text { Find }(u, \varphi) \in K(\Omega) \text { such that } \forall(v, \psi) \in K(\Omega), \\
\int_{\Omega} a_{\widehat{\varphi}}(u, v-u) d x+\int_{\Omega} a_{\widehat{\theta}}(\varphi, v-u) d x \geq \int_{\Omega} \frac{f}{2 h}(v-u) d x, \\
\int_{\Omega} \Delta \varphi \Delta \psi d x-E \int_{\Omega} a_{\widehat{\theta}}(u, \psi) d x=0 .
\end{array}\right.
$$

Lemma 3.3. For all $\widehat{\varphi} \in C^{2}(\Omega), \widehat{\theta} \in C^{2}(\Omega)$ with $D_{2} \widehat{\varphi}$ satisfying Assumption 3.2 , problem (3.3) has a unique solution $(u, \varphi)$ in $K(\Omega)$.

Proof. It is given in Appendix A.

Remark 3.4. General results about variational inequality have established that the solution to the obstacle problem for a second-order linear elliptic operator in a smooth domain $\Omega$ belongs to $\left.C^{1+\alpha}(\Omega), \alpha \in\right] 0,1[$, (see e.g., [10] or [23]). Our next work [4] will consist in extending this result to the case of problem (2.4) under the assumptions of Lemma 3.3. The extended proof of this intermediate result will be presented separately.

Let us in addition give a regularity statement, which although remaining a conjecture, is a very important founding principle of what follows.

Conjecture 3.5. Assume all the data are $C^{\infty}$, then the free boundary in the solution to problem (2.4) is piecewise $C^{\infty}$. 
Using the regularity results formulated in Remark 3.4 and Conjecture 3.5, problem (3.2) is now well-posed:

$$
\begin{cases}-[u, \widehat{\varphi}]-[\varphi, \widehat{\theta}]=\frac{f}{2 h} & \text { in } \Omega / I, \\ \Delta^{2} \varphi+E[u, \widehat{\theta}]=0 & \text { in } \Omega, \\ u>0 & \text { in } \Omega / I, \\ u=0 & \text { in } I, \\ \partial_{n} u=0 & \text { on } \partial I, \\ u=1, \quad \varphi=0, \quad \partial_{n} \varphi=0 & \text { on } \partial \Omega .\end{cases}
$$

Remark 3.6. In addition to being positive in $\Omega$, the component $u$ of the solution to problem (3.4) should be equal to zero in an unknown subdomain $I$, this explains the addition of the condition $\partial_{n} u=0$ on $\partial I$. Basically, this condition results from the Euler-Lagrange equations associated with problem (2.4) and is a characteristics of free boundary problems. Since the derivatives of $u$ appear only at the secondorder in the boundary value problem (3.4), it might seem that too many conditions are required for this problem to have a solution, but in fact the additional matching condition is exactly the closure condition which is needed since the free boundary $\partial I$ is unknown. The same kind of matching condition, but at higher order, had been proved in [3] and used in [7] in the case of the biharmonic operator.

Comment. (i) Conjecture 3.5 is known as Schaeffer's conjecture. It has been stated for the first time in [19]. It is optimal in the sense that one cannot expect the free boundary to be $C^{\infty}$ in general. As a matter of fact, even with extremely smooth data, singularities may appear on the free boundary. In particular, explicit examples of the occurrence of cusps have been presented in [22].

(ii) The free boundary is actually piecewise $C^{\infty}$, with singular points of the free boundary strictly separated. The smoother the data $f$ (remember that problem (2.4) has homogeneous or constant boundary conditions and that the obstacle is flat), the smoother the free boundary is between adjacent singular points [9]; moreover, in the particular case of the harmonic operator and a constant force, lower bounds have been given on the distance between two consecutive singular points in a particular case [17], together with upper bounds on the number of singularities depending on the size of the domain.

(iii) From the topological point of view, it has only been observed in the case of the harmonic operator that strong convexity conditions are required for the free boundary to be a Jordan curve.

\subsection{A simple particular case}

This short section aims at beginning to understand the difference with the scalar case, and to observe that, for some particular examples, problems (3.1) or (3.4) are 
not very far from those previously studied, regardless the fourth-order term and the coupling of the unknowns. As a matter of fact, let us look at a simple academic case. This is a very simple problem, with special data and boundary conditions in such a way that a decoupling procedure can be performed.

Assume the map $\widehat{\theta}$ is such that $D_{2}(\widehat{\theta})=I d$ which implies $[\psi, \widehat{\theta}]=\Delta \psi,[v, \widehat{\theta}]=$ $\Delta v$, and choose boundary conditions $\psi=\Delta \psi=0$ on $\partial \Omega\left(\right.$ instead of $\psi=\partial_{n} \psi=0$ on $\partial \Omega$ ). Using these simplifications, problem (3.4) amounts to solving:

Find $(v, \psi)$ in appropriate spaces and a smooth enough subdomain $I$ such that:

$$
\begin{cases}-[v, \widehat{\varphi}]-\Delta \psi=f / 2 h & \text { in } \Omega / I, \\ \Delta(\Delta \psi+E v)=0 & \text { in } \Omega, \\ v=1, & \text { on } \partial \Omega, \\ v=0, \quad \partial_{n} v=0 & \text { on } \partial I, \\ \psi=\Delta \psi=0 & \text { on } \partial \Omega .\end{cases}
$$

It is easily seen that this problem can be uncoupled by introducing the auxiliary unknown $\zeta:=\Delta \psi+E v$ and solved in three steps as follows.

Step 1. Find $\zeta$ in $\Omega$ solution to

$$
\begin{cases}\Delta \zeta=0 & \text { in } \Omega, \\ \zeta=E & \text { on } \partial \Omega .\end{cases}
$$

Step 2. Find $v$ in $\Omega / I$ solution to

$$
\begin{cases}-[v, \widehat{\varphi}]+E v=f / 2 h+\zeta & \text { in } \Omega / I, \\ v=0, \quad \partial_{n} v=0 & \text { on } \partial I, \\ v=1 & \text { on } \partial \Omega .\end{cases}
$$

Step 3. Find $\psi$ in $\Omega$ solution to

$$
\begin{cases}\Delta \psi=\zeta-E v & \text { in } \Omega, \\ \psi=0 & \text { on } \partial \Omega .\end{cases}
$$

Step 1 is an elementary Dirichlet problem for the harmonic operator in a smooth domain, the solution of which is as smooth as we want. Then, under the assumption $D_{2} \widehat{\varphi}>0$ to ensure the coercivity, step 2 is a boundary value problem in $\Omega / I$ for a second-order coercive operator, a smooth right-hand side, and overmuch boundary conditions. It is exactly the reformulation of the obstacle problem studied by Schaeffer in [21, Sec. 1, Problem 1.1] which has a solution in $C^{1+\varepsilon}$. Step 3 again is a Dirichlet problem for the harmonic operator with a smooth enough right-hand side.

In other words, due to the very special map $\widehat{\theta}$ and to the abstract boundary conditions, the initial coupled problem splits into two second-order scalar bilateral problems for unknowns $\zeta$ and $\psi$ posed in the whole domain $\Omega$ and an obstacle problem for the unknown $v$ in the same domain $\Omega$. The same idea has been used 
in [13]. Keeping this idea of uncoupling in the general case would involve Green's operator which might lead to additional complexity, so that we keep the whole operator with the vectorial unknown in the sequel, but it was interesting to observe that in particular cases the obstacle problem in the vectorial case turns out to be of Schaeffer's kind.

\section{The Stability Result}

We consider the shallow weakly curved membrane under study submitted to an external force $f^{0} \in C^{\infty}(\bar{\Omega})$ normal to the horizontal plane and we denote by $\left(u\left(f^{0}\right), \varphi\left(f^{0}\right)\right)$ the unique solution of problem (3.4) associated with the coincidence set $I\left(f^{0}\right)$. We now present the main result of the paper which is the following theorem.

Theorem 4.1. Assume that:

(i) $-f^{0} \geq \delta_{0}>0$ in $I\left(f^{0}\right)$;

(ii) $\partial I\left(f^{0}\right)$ is a $C^{\infty}$ Jordan curve.

Then for any $f$ sufficiently close to $f^{0}$ in $C^{\infty}(\bar{\Omega})$, the coincidence set $I(f)$ associated with the solution $(u(f), \varphi(f))$ is such that $\partial I(f)$ is also $C^{\infty}$ and diffeomorphic to $\partial I\left(f^{0}\right)$.

\subsection{The steps of the proof}

Up to the end of this work, the solution $\left(u\left(f^{0}\right), \varphi\left(f^{0}\right), I\left(f^{0}\right)\right)$ will be denoted by $\left(u^{0}, \varphi^{0}, I^{0}\right)$ for simplicity, and the free boundary $\partial I\left(f^{0}\right)$ by $\Gamma^{0}$. Assume $I^{0}$ is smooth and simply connected. It is clear from elementary examples that this assumption can be satisfied. As a complement to Schaeffer's conjecture, it will be taken as a consequence of the weak curvature. The proof of Theorem 4.1 is relatively long. It is broken into several steps which we summarize for the sake of clarity:

(1) The first step consists in moving the curve $\Gamma^{0}$ in the plane of the obstacle in order to obtain a new curve of the plane which we denote by $\Gamma^{\eta}$ using a smooth and small enough real function $\eta$ defined on $\Gamma^{0}$. If $\Gamma^{0}$ is a smooth Jordan curve, then $\Gamma^{\eta}$ will also be a smooth Jordan curve, close to $\Gamma^{0}$, so that $\Gamma^{\eta}$ will be taken as the boundary of a new smooth subdomain $I^{\eta}$ in the plane of the obstacle.

(2) We choose a force $f$ in some neighborhood of $f^{0}$ in $C^{\infty}(\Omega)$, and we build a bilateral problem in $\Omega$ with the same left-hand side as (2.4), the external force $f$ in $\Omega / I^{\eta}$, and the component $u^{\eta}$ of the solution satisfying $u^{\eta}=0$ in $I^{\eta}$. This problem is shown to have a single sufficiently smooth solution. Two conditions are necessary for this solution to be the solution to the unilateral problem (2.4) with $\partial I^{\eta}=\Gamma^{\eta}$ as the boundary of the coincidence set:

- the matching condition $u^{\eta}=\partial_{n} u^{\eta}=0$ on $\Gamma^{\eta}$ presented in problem (3.4) must be satisfied, 
- the component $u^{\eta}$ of this solution must be positive in $\Omega / I^{\eta}$, which means that the membrane must be above the obstacle.

(3) We calculate the trace of the normal derivative of this function $u$ on the smooth curve $\Gamma^{\eta}$. This procedure defines an operator denoted by $\mathcal{T}(\eta, f)$ which, with any pair $(\eta, f)$, associates the normal derivative of the component $u^{\eta}$ of the solution along a curve of the plane of the obstacle. Two qualitative properties of operator $\mathcal{T}$ stem from this definition:

- $\mathcal{T}$ is a nonlocal operator, of the type of Dirichlet to Neumann operator;

- $\mathcal{T}(\eta, f)$ is such that $\mathcal{T}\left(0, f^{0}\right)=0$, which recalls that $\Gamma^{0} \equiv \partial I^{0}\left(f^{0}\right)$ is the free boundary associated with the solution for $f=f^{0}$.

(4) We solve the implicit equation $\mathcal{T}(\eta, f)=0$, in order to obtain $\eta$ as a function of $f$ in a neighborhood of $\left(\eta=0, f=f^{0}\right)$. This will be done by using Nash-Moser Implicit Function Theorem.

(5) We verify that the solution $\left(u^{\eta}(f), \varphi^{\eta}(f)\right)$ is such that $u^{\eta}(f)>0$ in $\Omega / I^{\eta}$.

\subsection{Nash-Moser Theorem}

The main tool for the proof of Theorem 4.1 is Nash-Moser Implicit Function Theorem (exposed in $[1,8,24])$. Let $\sigma>2$ and let $\eta(s) \in C^{\sigma}\left(\Gamma^{0}\right)$ and $f \in C^{\sigma}(\bar{\Omega})$ be two real functions. We define the vector space

$$
\mathcal{W}=C^{\sigma}\left(\Gamma^{0}\right) \times C^{\sigma}(\bar{\Omega})
$$

with a norm given by

$$
\|(u, f)\|_{\lambda}:=\|u\|_{\lambda}+\|f\|_{\lambda}, \forall \lambda>0,
$$

and introduce the subset of sufficiently small functions:

$$
\widetilde{\mathcal{W}}=\left\{(\eta, f) \in \mathcal{W} \backslash\|(u, f)\|_{\sigma+k}<\varepsilon, k \geq 0\right\} .
$$

Let us now state the following theorem.

Theorem 4.2 (Nash-Moser Implicit Function Theorem). Assume an operator $\mathcal{T}(\cdot, \cdot)$ is such that

$$
\left\{\begin{array}{c}
\text { (i) } \mathcal{T}(\eta, f) \text { is a nonlinear operator of order } m \text { from } \widetilde{\mathcal{W}} \text { to } C^{\sigma-m}\left(\Gamma^{0}\right), \\
\quad \text { satisfying } \mathcal{T}\left(0, f_{0}\right)=0, \text { and there exist four positive } \\
\quad \text { constants } C_{1}, C_{2}, C_{3}, C_{4} \text { such that } \\
\text { (ii) }\|\mathcal{T}(\eta, f)\|_{\lambda} \leq C_{1}\left(\|(\eta, f)\|_{\lambda+m}+1\right) \text { for any } \lambda \geq \sigma, \\
\text { (iii) }\left\|\mathcal{T}\left(\eta, f_{1}\right)-\mathcal{T}\left(\eta, f_{2}\right)\right\|_{\sigma-m} \leq C_{2}\left\|f_{1}-f_{2}\right\|_{\sigma}, \\
\text { (iv) } \quad \exists \mathcal{L}_{\eta, f} \in \mathbb{L}\left(C^{\sigma}, C^{\sigma-m}\right) \text { such that } \\
\quad\left\|\mathcal{T}(\eta+\zeta, f)-\mathcal{T}(\eta, f)-\mathcal{L}_{\eta, f} \zeta\right\|_{\sigma-m} \leq C_{3}\left(\|\mathcal{T}(\eta, f)\|_{\sigma}\|\zeta\|_{\sigma}+\|\zeta\|_{\sigma}^{2}\right), \\
\text { (v) } \quad \exists \ell_{\eta, f} \in \mathbb{L}\left(C^{\sigma+m}\left(\Gamma^{\eta}\right), C^{\sigma}\left(\Gamma^{\eta}\right)\right) \text { such that } \forall(\eta, f) \in \widetilde{\mathcal{W}}, \\
\mathcal{L}_{\eta, f}\left(\ell_{\eta, f}(\zeta)\right)=\zeta \text { and }\left\|\ell_{\eta, f}\right\|_{\lambda} \leq C_{4}\left(\|\eta\|_{0}\|(\eta, f)\|_{\lambda+m}\right) .
\end{array}\right.
$$


Then there exist $\varepsilon>0$ and $\kappa \geq \lambda$ such that for all $f$ with $\|f\|_{\kappa} \leq \varepsilon$, the equation $\mathcal{T}(\eta, f)=0$ defines a unique function $\eta=\eta(f)$.

\section{Remark 4.3.}

- We shall prove that Theorem 4.2 applies in the case of problem (2.4) in a neighborhood of the solution associated with the force $f^{0} \in C^{\infty}(\bar{\Omega})$, for $m=1, \sigma=2+\alpha$ with $0<\alpha<1$.

- Comments on assumptions (i) to (v):

- assumption (i) means that operator $\mathcal{T}$ involves a loss of derivatives (exactly $m$ orders of derivation), and is the reason for which the classical implicit function theorem does not apply,

- assumption (ii) means that $\mathcal{T}$ is a "good" or "tame" mapping: such an estimate means that $\mathcal{T}$ should not increase too fast,

- assumption (iii) means that $\mathcal{T}$ should be continuous with respect to the forces,

- assumption (iv) means that $\mathcal{L}_{\eta, f}$ approximates the derivative of $\mathcal{T}$ with respect to $\eta$ at $(\eta, f)$,

- assumption (v) says that operator $\mathcal{L}_{\eta, f}$ has a bounded inverse.

- The main qualitative differences between classical Implicit Function Theorem and Nash-Moser Implicit Function Theorem are given by assumptions (iv) and (v): operator $\mathcal{L}$ is not the Fréchet derivative of $\mathcal{T}$ at $\left(0, f^{0}\right)$, which would be required by Implicit Function Theorem, but an approximation of the derivative of $\mathcal{T}$ which must be invertible in a whole neighborhood of $\left(0, f^{0}\right)$.

\section{Proof of the Stability Theorem}

The remaining part of the work is devoted to the proof of the main result.

\subsection{Construction of operator $\mathcal{T}(\eta, f)$}

\subsubsection{Moving the free boundary}

We assume that for a smooth force $f^{0} \in C^{\infty}(\bar{\Omega})$, the solution involves a coincidence set $I^{0}$ the boundary of which $\Gamma^{0}$ is a smooth Jordan curve according to Conjecture 3.5. Let $s$ be a curvilinear abscissa on $\Gamma^{0}$ and introduce a real-valued function $\eta \in C^{k}\left(\Gamma^{0}\right),\|\eta\|_{k} \ll 1$. We define a map

$$
\phi^{\eta}: \Gamma^{0} \rightarrow \mathbb{R}^{2}, \quad \phi^{\eta}(s)=s+\eta(s) N^{0}(s),
$$

where $N^{0}$ is the unit normal to $\Gamma^{0}$ in the plane of the obstacle directed outwards to $I^{0}$.

Let $\Gamma^{\eta}$ be the image of $\Gamma^{0}$ by $\phi^{\eta}$. Due to the smallness and the regularity of function $\eta$, we deduce first that $\Gamma^{\eta}$ is also a smooth Jordan curve, which is nothing but the classical injectivity condition and we denote by $I^{\eta}$ the smooth domain enclosed by $\Gamma^{\eta}$, and second that $\Gamma^{\eta} \cap \partial \Omega=\emptyset$. Since $\Omega / I^{0}$ is diffeomorphic to an annulus, $\Omega / I^{\eta}$ is consequently also diffeomorphic to an annulus. Choosing 
$\eta \in C^{\infty}\left(\Gamma^{0}\right)$ the diffeomorphism will be $C^{\infty}$, which will give a good framework for the application of Nash-Moser Theorem.

\subsubsection{An intermediate bilateral problem, and the} construction of the implicit equation

We now use the above construction of the smooth subdomain $I^{\eta}$ of $\Omega$ and state the following result.

Lemma 5.1. Let $\Omega$ be a bounded smooth domain of the plane, $I^{\eta}$ a given smooth subdomain of $\Omega$ with boundary $\Gamma^{\eta}$ satisfying $\Gamma^{\eta} \cap \partial \Omega=\emptyset$, let some loading $f \in$ $C^{\infty}(\Omega)$ be applied on $\Omega$ and use the notations of problem (3.4), then the following bilateral coupled problem has a single solution $(u, \varphi)$

$$
\begin{cases}-[u, \widehat{\varphi}]-[\varphi, \widehat{\theta}]=\frac{f}{2 h} & \text { in } \Omega / I^{\eta}, \\ \Delta^{2} \varphi+E[u, \widehat{\theta}]=0 & \text { in } \Omega, \\ u=0 & \text { in } I^{\eta}, \\ u=1, \quad \varphi=0, \quad \partial_{n} \varphi=0 & \text { on } \partial \Omega .\end{cases}
$$

Remark. The component $u$ of the solution to problem (5.2) is as smooth as necessary in $\overline{\Omega / I^{\eta}}$, so that the normal derivative $\partial_{n} u$ on $\Gamma^{\eta}$ will also be as smooth as required.

The proof of the existence of a single solution is very closed to that of Lemma 3.3, the details of which are given in Appendix A. The only difference, which does not change the result, since we are dealing with a linear operator, is that the inequality in the closed convex $K(\Omega)$ is changed into an equality in the following closed convex:

$$
\mathbb{K}=\left\{(v, \psi) \in H^{1}(\Omega) \times H_{0}^{2}(\Omega), v=0 \text { in } I^{\eta}, v=1 \text { on } \partial \Omega\right\} .
$$

The part of this result concerning regularity will be investigated in [4].

\subsection{The assumptions of Nash-Moser Theorem are satisfied}

We first observe that assumptions (i) to (iii) of Theorem 4.2 actually hold. As a matter of fact, assumption (i) follows directly from the construction of operator $\mathcal{T}$, and assumptions (ii) and (iii) result from Shauder estimates which have been used exactly in the same framework in [21] and more recently in [14].

But the remaining assumptions (iv) and (v) must be studied carefully. By comparison with the previous works, we focus on the difference which arises from the coupling between the components. According to point (3) of Sec. 4.1, operator $\mathcal{T}$ is defined as

$$
\mathcal{T}:(\eta, f) \rightarrow \mathcal{T}(\eta, f)=D u \circ \phi^{\eta}=\left(\nabla u \cdot N^{0}\right) \circ \phi^{\eta}
$$




\subsubsection{Estimating the derivative of $\mathcal{T}$}

We shall use the notation $D \mathcal{T}$ to denote the derivative of operator $\mathcal{T}$ with respect to its first variable $\eta$. In the plane of the obstacle we introduce a tubular neighborhood $\mathcal{V}\left(\Gamma^{\eta}\right)$ of the curve $\Gamma^{\eta}$ defined for small enough $\left|t_{0}\right|$ by:

$$
\mathcal{V}\left(\Gamma^{\eta}\right)=\left\{s^{0}+(\eta(s)+t) N^{0}, s^{0} \in \Gamma^{0},-t_{0}<t<t_{0}\right\} .
$$

We now consider problem (5.2) with a given external force $f$, introduce a realvalued function $\zeta$ defined on $\Gamma^{0}$ of the same smoothness as $\eta$ in such a way that the curve $\Gamma^{\eta+\varepsilon \zeta}$ belongs to $\mathcal{V}\left(\Gamma^{\eta}\right)$ for $\varepsilon$ small enough. Let $I^{\eta+\varepsilon \zeta}$ be the corresponding compact subdomain with boundary $\Gamma^{\eta+\varepsilon \zeta}$.

Let us recall that the pair $(u, \varphi)$ is the solution to problem (5.2) from which $\mathcal{T}(\eta, f)$ has been defined, and introduce the solution $(u+\varepsilon v, \varphi+\varepsilon \psi)$ to the following perturbed problem associated with the curve $\Gamma^{\eta+\varepsilon \zeta}$ :

$$
\begin{cases}-[u+\varepsilon v, \widehat{\varphi}]-[\varphi+\varepsilon \psi, \widehat{\theta}]=\frac{f}{2 h} & \text { in } \Omega / I^{\eta+\varepsilon \zeta}, \\ \Delta^{2}(\varphi+\varepsilon \psi)+E[u+\varepsilon v, \widehat{\theta}]=0 & \text { in } \Omega, \\ u+\varepsilon v=0 & \text { in } I^{\eta+\varepsilon \zeta,} \\ u+\varepsilon v=1, \quad \varphi+\varepsilon \psi=0, \quad \partial_{n}(\varphi+\varepsilon \psi)=0 & \text { on } \partial \Omega .\end{cases}
$$

The derivative of $\mathcal{T}$ with respect to $\eta$ is given by $\lim _{\varepsilon \rightarrow 0} \frac{\mathcal{T}(\eta+\varepsilon \zeta, f)-\mathcal{T}(\eta, f)}{\varepsilon \zeta}$ that we can compute from the solutions to problems (5.2) and (5.4). The main step is the following result.

Theorem 5.2. Let $\alpha^{\eta}$ be the angle between $N^{0}$ and $N^{\eta}$ the outer normal to $\Gamma^{\eta}$. The derivative of $\mathcal{T}$ is approximated by:

$$
D \mathcal{T}(\eta, f) \approx-\frac{\cos ^{2} \alpha^{\eta}}{\left\|N^{\eta}\right\|_{\widehat{\varphi}}^{2}}\left(\frac{f}{2 h}+[\varphi, \widehat{\theta}]\right) \quad \text { on } \Gamma^{\eta} .
$$

The computation of this derivative is broken into two steps. In Lemma 5.3 we prove that an approximation of the derivative of the operator $\mathcal{T}$ can be given by the second-order derivative of $u$ along the normal $N^{0}$ and in Lemma 5.4 we compute the expression of the second-order normal derivative along $N^{\eta}$.

Lemma 5.3. Assume $u=0$ and $\mathcal{T}(\eta, f)=0$ on $\Gamma^{\eta}$, then the derivative to $\mathcal{T}$ can be approximated in the following way

$$
D \mathcal{T}(\eta, f) \approx D^{2} u \quad \text { on } \Gamma^{\eta}
$$

Proof. First step. We first compute $u$ in the tubular neighborhood of $\Gamma^{\eta}$ defined by Eq. (5.3). 
Let $s^{0}$ be a point on $\Gamma^{0}$ and $s^{\eta}$ be a point on $\Gamma^{\eta}$ (with $\eta=\eta\left(s^{0}\right)$ ). We consider the Taylor expansion of $u$ around $s^{\eta}$, thus for $s^{\eta+t}=s^{0}+\left(\eta\left(s^{0}\right)+t\right) N^{0}=s^{0}+$ $\eta\left(s^{0}\right) N^{0}+t N^{0}=s^{\eta}+t N^{0}$ we get

$$
u\left(s^{\eta+t}\right)=u\left(s^{\eta}\right)+t D u\left(s^{\eta}\right)+\frac{1}{2} t^{2} D^{2} u\left(s^{\eta}\right)+\cdots,
$$

where $D u\left(s^{\eta}\right)=\lim _{t \rightarrow 0} \frac{u\left(s^{\eta+t}\right)-u\left(s^{\eta}\right)}{t}=\left(\nabla u \cdot N^{0}\right) \circ \phi_{\eta}$ is the Gâteaux derivative of $u$ on $\Gamma^{\eta}$ in the direction of $N^{0}$ and $D^{2} u\left(s^{\eta}\right)=\nabla^{2} u\left(N^{0}, N^{0}\right) \circ \phi_{\eta}$ with $\nabla u=\left(\frac{\partial u}{\partial x_{1}}, \frac{\partial u}{\partial x_{2}}\right)$ at the point $s^{0}$. On $\Gamma^{\eta}$ we assume $u=0$ and $\mathcal{T}(\eta, f)=D u \circ \phi_{\eta}=\left(\nabla u \cdot N^{0}\right) \circ \phi_{\eta}=0$, hence it remains:

$$
u\left(s^{\eta+t}\right)=\frac{1}{2} t^{2} D^{2} u\left(s^{\eta}\right)+\cdots
$$

Second step. Using a smooth function $\zeta$ we move the boundary from $\Gamma^{\eta}$ to $\Gamma^{\eta+\varepsilon \zeta}$ in $\mathcal{V}\left(\Gamma^{\eta}\right)$, and we aim at calculating the problem of which the perturbation due to this change is the solution. Let us rewrite the bilateral problems (5.2) and (5.4) respectively for given internal boundary $\Gamma^{\eta}$ and $\Gamma^{\eta+\varepsilon \zeta}$ as

$$
\begin{cases}-[u, \widehat{\varphi}]-[\varphi, \widehat{\theta}]=\frac{f}{2 h} & \text { in } \Omega / I^{\eta} \\ \Delta^{2} \varphi+E[u, \widehat{\theta}]=0 & \text { in } \Omega / I^{\eta} \\ u=0, \quad \Delta^{2} \varphi=0 & \text { in } I^{\eta} \\ u=1, \quad \varphi=0, \quad \partial_{n} \varphi=0 & \text { on } \partial \Omega\end{cases}
$$

and

$$
\begin{cases}-[u+\varepsilon v, \widehat{\varphi}]-[\varphi+\varepsilon \psi, \widehat{\theta}]=\frac{f}{2 h} & \text { in } \Omega / I^{\eta+\varepsilon \zeta}, \\ \Delta^{2}(\varphi+\varepsilon \psi)+E[u+\varepsilon v, \widehat{\theta}]=0 & \text { in } \Omega / I^{\eta+\varepsilon \zeta}, \\ u+\varepsilon v=0, \quad \Delta^{2}(\varphi+\varepsilon \psi)=0 & \text { in } I^{\eta+\varepsilon \zeta}, \\ u+\varepsilon v=1, \quad \varphi+\varepsilon \psi=0, \quad \partial_{n}(\varphi+\varepsilon \psi)=0 & \text { on } \partial \Omega .\end{cases}
$$

The purpose of this step is to establish that $(v, \psi) \approx(0,0)$ at the order $\varepsilon$. In order to do that we consider the two simpler cases: first, $I^{\eta} \subset I^{\eta+\varepsilon \zeta} \subset \Omega$, and then $I^{\eta+\varepsilon \zeta} \subset I^{\eta}$ (i.e. function $\zeta$ defined on $\Gamma^{0}$ is first assumed to be positive everywhere, then negative everywhere). The general case will then be easily deduced.

- The case $I^{\eta} \subset I^{\eta+\varepsilon \zeta} \subset \Omega$ implies $\Omega / I^{\eta+\varepsilon \zeta} \subset \Omega / I^{\eta}$ and we get:

$$
\begin{cases}-[v, \widehat{\varphi}]-[\psi, \widehat{\theta}]=0 & \text { in } \Omega / I^{\eta+\varepsilon \zeta} \\ u+\varepsilon v=0 & \text { in } I^{\eta+\varepsilon \zeta} / I_{\eta} \\ v=0 & \text { in } I^{\eta} \\ \Delta^{2} \psi+E[v, \widehat{\theta}]=0 & \text { in } \Omega \\ v=0, \quad \psi=0, \quad \partial_{n} \psi=0 & \text { on } \partial \Omega\end{cases}
$$


We compute $\psi$ in terms of $v$ through the equations $\Delta^{2} \psi+E[v, \widehat{\theta}]=0$ in $\Omega / I^{\eta}$ and $\Delta^{2} \psi=0$ in $I^{\eta}$ with homogeneous boundary conditions on $\partial \Omega$, then we compute $v$ from the equation $-[v, \widehat{\varphi}]-[\psi, \widehat{\theta}]=0$ in $\Omega / I^{\eta+\varepsilon \zeta}$ with the boundary condition $u+\varepsilon v=0$ on $\Gamma^{\eta+\varepsilon \zeta}$. Since $u$ is of order $\varepsilon^{2}$ on $\partial I^{\eta+\varepsilon \zeta}$ due to (5.5), we get that $(v, \psi)$ is the solution of a homogeneous problem with homogeneous boundary conditions and therefore $(v, \psi) \approx(0,0)$.

- The case $I^{\eta+\varepsilon \zeta} \subset I^{\eta}$ implies $\Omega / I^{\eta} \subset \Omega / I^{\eta+\varepsilon \zeta}$ and we get:

$$
\begin{cases}-[v, \widehat{\varphi}]-[\psi, \widehat{\theta}]=0 & \text { in } \Omega / I^{\eta}, \\ -[v, \widehat{\varphi}]-[\psi, \widehat{\theta}]=\frac{1}{\varepsilon}(f+[\varphi, \widehat{\theta}]) & \text { in } I^{\varepsilon}:=I^{\eta} / I^{\eta+\varepsilon \zeta}, \\ v=0 & \text { in } I^{\eta+\varepsilon \zeta}, \\ \Delta^{2} \psi+E[v, \widehat{\theta}]=0 & \text { in } \Omega, \\ v=0, \quad \psi=0, \quad \partial_{n} \psi=0 & \text { on } \partial \Omega .\end{cases}
$$

Let $F=\max _{I^{\varepsilon}}\|(f+[\varphi, \widehat{\theta}])\|$ and let us recall that Area $\mathrm{I}^{\varepsilon} \approx \varepsilon$. Then the weak solution of system (5.7) is bounded as

$$
\begin{aligned}
\|\Delta \psi\|^{2}+\|\nabla v\|^{2} & =E \int_{I^{\varepsilon}} \frac{1}{\varepsilon} v(f+[\varphi, \widehat{\theta}]) d x \\
& \leq C_{1} E F\|v\| \Rightarrow\|[\psi, \widehat{\theta}]\| \leq C_{2},
\end{aligned}
$$

where $C_{1}>0, C_{2}>0$ depend upon the data $(E, \Omega, \widehat{\theta}, \widehat{\psi})$ but are independent of $\varepsilon$. The displacement $v$ is therefore solution to the problem

$$
\begin{cases}-[v, \widehat{\varphi}]-[\psi, \widehat{\theta}]=0 & \text { in } \Omega / I^{\eta}, \\ -[v, \widehat{\varphi}]-[\psi, \widehat{\theta}]=\frac{1}{\varepsilon}(f+[\varphi, \widehat{\theta}]) & \text { in } I^{\varepsilon}=I^{\eta} / I^{\eta+\varepsilon \zeta}, \\ v=0 & \text { in } I^{\eta+\varepsilon \zeta},\end{cases}
$$

from which a direct computation gives $v \approx \varepsilon$ in $I^{\varepsilon}$ which in turn gives the result.

Lemma 5.4. For any $F \in L^{2}(\Omega)$, let us consider the following boundary value problem with mixed boundary conditions

$$
\begin{cases}-[u, \widehat{\varphi}]=F & \text { in } \Omega / I^{\eta} \\ u=0 & \text { on } \partial \Omega \\ \partial_{n} u=\mathcal{T} & \text { on } \Gamma^{\eta}\end{cases}
$$

Then the second-order normal derivative $\partial_{n n} u$ along the normal $N^{\eta}$ to $\Gamma^{\eta}$ is given by

$$
\partial_{n n} u=-F \frac{1}{\left\|N^{\eta}\right\|_{\widehat{\varphi}}^{2}} \quad \text { on } \Gamma^{\eta}
$$

with $\left\|N^{\eta}\right\|_{\widehat{\varphi}}^{2}=\left(N^{\eta}\right)^{t} D_{2} \widehat{\varphi} N^{\eta}$ where $D_{2} \widehat{\varphi}$ has already been defined as $\left(\begin{array}{cc}+\partial_{22} \widehat{\varphi} & -\partial_{12} \widehat{\varphi} \\ -\partial_{12} \widehat{\varphi} & +\partial_{11} \widehat{\varphi}\end{array}\right)$. 
Proof. We express $\partial_{i j} u$ on $\Gamma^{0}$ in terms of $\partial_{t} u$ and $\partial_{n} u$ and denote $N^{\eta}=\left(n_{1}, n_{2}\right)$, then we get

$$
\begin{aligned}
{[u, \widehat{\varphi}]=} & \partial_{n n} u\left(n_{1}^{2} \partial_{22} \widehat{\varphi}+n_{2}^{2} \partial_{11} \widehat{\varphi}-2 n_{1} n_{2} \partial_{12} \widehat{\varphi}\right) \\
& +\partial_{t n} u\left(-2 n_{1} n_{2} \partial_{22} \widehat{\varphi}+2 n_{1} n_{2} \partial_{11} \widehat{\varphi}+2\left(n_{1}^{2}-n_{2}^{2}\right) \partial_{12} \widehat{\varphi}\right) \\
& +\partial_{t t} u\left(n_{2}^{2} \partial_{22} \widehat{\varphi}+n_{1}^{2} \partial_{11} \widehat{\varphi}+2 n_{1}^{2} \partial_{12} \widehat{\varphi}\right) .
\end{aligned}
$$

Since $u=0$ on $\Gamma^{0}$ implies $\partial_{t t} u=\partial_{t n} u=0$ on $\Gamma^{0}$, it remains

$$
[u, \widehat{\varphi}]=\partial_{n n} u\left(n_{1}^{2} \partial_{22} \widehat{\varphi}+n_{2}^{2} \partial_{11} \widehat{\varphi}-2 n_{1} n_{2} \partial_{12} \widehat{\varphi}\right)=-F \quad \text { on } \Gamma^{\eta}
$$

from which (since $D_{2} \widehat{\varphi}$ is positive definite) we can compute $\partial_{n n} u$ on $\Gamma^{\eta}$ and get the result.

The proof of Theorem 5.2 is then completed by gathering Lemmas 5.3 and 5.4 along with the remark that:

$$
\partial_{n n} u \circ \phi_{\eta}=\left(\left(N^{\eta}\right)^{t} D^{2} u N^{\eta}\right) \circ \phi_{\eta}=\cos ^{2} \alpha_{\eta}\left(\left(N^{0}\right)^{t} D^{2} u N^{0}\right) \circ \phi_{\eta} .
$$

The next lemma deals with geometrical considerations on the free boundary in order to get an appropriate bound on $D \mathcal{T}$. Let us recall that the smoothness of the free boundary $\Gamma^{0}$ is an assumption of Theorem 4.1 which results from Schaeffer's conjecture. Keeping this framework, and the smoothness of the diffeomorphism from $\Gamma^{0}$ to $\Gamma^{\eta}$, all the following calculations are justified.

Lemma 5.5. The angle of the 2 unit normals $\left(N^{0}, N^{\eta}\right)$ depends upon the curvature $\ddot{\gamma}$ of $\Gamma^{0}$, it is given by the expression:

$$
\frac{1}{\cos ^{2} \alpha_{\eta}}=1+\frac{\dot{\eta}^{2}}{(1-\eta \ddot{\gamma})^{2}}
$$

Moreover, for $|\eta|$ small enough there exists a positive constant $C$ such that:

$$
\frac{1}{\cos ^{2} \alpha_{\eta}} \leq C\left(1+\|\dot{\eta}\|_{C^{1}}\right)
$$

Proof. Let us consider a general parametrization: $t \in[0,1] \rightarrow(x(t), y(t))$. We get easily the components of the unit normal to $\Gamma^{0}, N^{0}=\left(\frac{-\dot{y}}{\sqrt{\dot{x}^{2}+\dot{y}^{2}}}, \frac{\dot{x}}{\sqrt{\dot{x}^{2}+\dot{y}^{2}}}\right)$ with the notation $\dot{x}=\frac{d x}{d t}, \dot{y}=\frac{d y}{d t}$. The components of a generic point on $\Gamma^{\eta}$ are $\left(x-\eta \frac{\dot{y}}{\sqrt{\dot{x}^{2}+\dot{y}^{2}}}, y+\eta \frac{\dot{x}}{\sqrt{\dot{x}^{2}+\dot{x}^{2}}}\right)$ and the components of the unit normal $N^{\eta}$ associated to this point read

$$
\begin{aligned}
N^{\eta}= & \frac{\left(\dot{x}^{2}+\dot{y}^{2}\right)}{|\eta(\ddot{x} \dot{y}-\dot{x} \ddot{y})|}\left(-\dot{y}-\frac{\dot{\eta} \dot{x}+\eta \ddot{x}}{\sqrt{\dot{x}^{2}+\dot{x}^{2}}}+\frac{\eta \dot{x}(\dot{x} \ddot{x}+\dot{y} \ddot{y})}{\left(\dot{x}^{2}+\dot{y}^{2}\right)^{3 / 2}},\right. \\
& \left.\dot{x}-\frac{\dot{\eta} \dot{y}+\eta \ddot{y}}{\sqrt{\dot{x}^{2}+\dot{y}^{2}}}+\frac{\eta \dot{y}(\dot{x} \ddot{x}+\dot{y} \ddot{y})}{\left(\dot{x}^{2}+\dot{y}^{2}\right)^{3 / 2}}\right) .
\end{aligned}
$$


This yields $\frac{1}{\cos ^{2} \alpha_{\eta}}=1+\frac{\frac{\dot{\eta}^{2}}{\dot{x}^{2}+\dot{y}^{2}}}{\left(1-\eta \frac{\dot{x} \dot{y}-\dot{y}}{\left.\left(\dot{x}^{2}+\dot{y}^{2}\right)^{3 / 2}\right)^{2}}\right.}=1+\frac{\frac{\dot{\eta}^{2}}{\dot{x}^{2}+\dot{y}^{2}}}{(1-\eta \dot{\gamma})^{2}}$ where we introduced the curvature $\ddot{\gamma}(t)=\frac{\dot{x} \ddot{y}-\ddot{x} \dot{y}}{\left(\dot{x}^{2}+\dot{y}^{2}\right)^{3 / 2}}$. Moreover, in the case of a parametrization with respect to the arc length, i.e. with $\dot{x}^{2}+\dot{y}^{2}=1$ we get the intrinsic formulation $\frac{1}{\cos ^{2} \alpha_{\eta}}=$ $1+\frac{\dot{\eta}^{2}}{(1-\eta \ddot{\gamma})^{2}}$.

Let $0<\delta<1$, we note that for $|\eta|<\frac{\delta}{\max |\ddot{\gamma}|}$ then $\frac{\dot{\eta}^{2}}{(1-\eta \ddot{\gamma})^{2}} \leq C\|\dot{\eta}\|_{C^{1}}$ with $C=\frac{1}{(1-\delta)^{2}}$.

\subsubsection{Assumptions (iv) and (v) are satisfied}

In order to carry out the end of the proof that operator $\mathcal{T}$ fulfills assumptions (iv) and (v) of Theorem 4.2, several results are necessary.

Lemma 5.6. Assume the equilibrium of a linearly elastic shallow shell is given by problem (5.2). Assume in addition that the shell is weakly curved, with the meaning that the reference configuration is given by a smooth function $\widehat{\theta}$ which is sufficiently small together with its first and second derivatives. Then the derivative $D \mathcal{T}(\eta, f)$ is strictly positive.

Proof. From the variational formulation of problem (5.2) we get the following equalities

$$
\left\{\begin{array}{l}
\|\nabla u\|_{\widehat{\varphi}}^{2}+\langle\nabla u, \nabla \varphi\rangle_{\widehat{\theta}}=\left\langle\frac{f}{2 h}, u\right\rangle \\
\|\Delta \varphi\|^{2}-E\langle\nabla u, \nabla \varphi\rangle_{\widehat{\theta}}=0
\end{array}\right.
$$

from which we obtain

$$
E\|\nabla u\|_{\widehat{\varphi}}^{2}+\|\Delta \varphi\|^{2}=E\left\langle\frac{f}{2 h}, u\right\rangle \Rightarrow\left\{\begin{array}{l}
\|\nabla u\|_{\widehat{\varphi}}^{2} \leq\left|\frac{f}{2 h}\right|\|u\|, \\
\|\Delta \varphi\|^{2} \leq E\left|\frac{f}{2 h}\right|\|u\| .
\end{array}\right.
$$

Using Poincaré type inequalities we get the following bounds where the constants $C_{i}\left(\Omega^{\eta}\right)$ and $\alpha\left(\Omega^{\eta}, \widehat{\varphi}, \widehat{\theta}\right)$ only depend on $\eta$ and on the data $\left(\Omega^{\eta}, \widehat{\varphi}, \widehat{\theta}\right)$, and where $\Omega^{\eta}:=\Omega / I^{\eta}$ for simplicity:

$$
\left\{\begin{array}{l}
\|u\| \leq C_{0}\left(\Omega^{\eta}\right)\|\nabla u\|_{\widehat{\varphi}} \Rightarrow\|u\| \leq \alpha_{0}\left(\Omega^{\eta}, \widehat{\varphi}, \widehat{\theta}\right)\left|\frac{f}{2 h}\right|, \\
\|\Delta \varphi\|^{2} \leq E\left|\frac{f}{2 h}\right|\|u\| \Rightarrow\|\Delta \varphi\| \leq \alpha_{1}\left(\Omega^{\eta}, \widehat{\varphi}, \widehat{\theta}\right) \sqrt{E}\left|\frac{f}{2 h}\right| .
\end{array}\right.
$$

Poincaré type inequalities in $H_{0}^{2}$ give the equivalence between $\|\varphi\|_{H_{0}^{2}(\Omega)}$ and $\|\Delta \varphi\|_{0}$. From $\|[\varphi, \widehat{\theta}]\|_{0} \leq C(\widehat{\theta})\|\varphi\|_{H_{0}^{2}(\Omega)}$, where $C(\widehat{\theta}):=\max _{x \in \Omega}\left(\left|\partial_{11} \widehat{\theta}\right|,\left|\partial_{12} \widehat{\theta},\right| \partial_{22} \widehat{\theta}||\right)$, we obtain $\|[\varphi, \widehat{\theta}]\|_{0} \leq \alpha_{1} \widehat{\theta} \sqrt{E} \frac{f}{2 h}$. 
Going back to the first line of problem (5.2) rewritten as $-[u, \widehat{\varphi}]=\frac{f}{2 h}(1+$ $\left.[\varphi, \widehat{\theta}] \frac{2 h}{f}\right)$, we observe that $\left\|[\varphi, \widehat{\theta}] \frac{2 h}{f}\right\|$ will be small with respect to 1 if $C(\widehat{\theta})$ is small.

We can consequently write from Theorem 5.2

$$
D \mathcal{T}(\eta, f) \approx-\frac{\cos ^{2} \alpha_{\eta}}{\left\|N^{\eta}\right\|_{\widehat{\varphi}}^{2}} \frac{f}{2 h},
$$

which gives the result since assumption (i) of Theorem 4.1 implies that $f$ is strictly negative in $I^{0}$.

Assumption (iv) of Theorem 4.2 reads as follows.

Lemma 5.7. Let $(\eta, f) \in \widetilde{\mathcal{W}}$. There exists a linear operator $\mathcal{L}_{\eta, f} \in \mathbb{L}\left(\mathbb{C}^{\sigma}, \mathbb{C}^{\sigma-1}\right)$ such that for all $(\eta+\zeta, f) \in \widetilde{\mathcal{W}}$ we have the following bound:

$$
\left\|\mathcal{T}(\eta+\zeta, f)-\mathcal{T}(\eta, f)-\mathcal{L}_{\eta, f} \zeta\right\|_{\sigma-m} \leq C\left(\|\mathcal{T}(\eta, f)\|_{\sigma}\|\zeta\|_{\sigma}+\|\zeta\|_{\sigma}^{2}\right) .
$$

Proof. The proof involves three main steps, together with some technical points which are postponed to Appendix B. Let us first recall the notation $\mathcal{T}(\eta, f)=$ $D u \circ \phi_{\eta}=\left(\nabla u \cdot N_{0}\right) \circ \phi_{\eta}$ and split up the left-hand side of (5.9) into the sum of four terms:

$$
\left\{\begin{aligned}
\mathcal{T}(\eta+ & \zeta, f)-\mathcal{T}(\eta, f)-\mathcal{L}_{\eta, f} \zeta \\
= & D u^{\zeta} \circ \phi_{\eta+\zeta}-D u \circ \phi_{\eta}-\mathcal{L}_{\eta, f} \zeta \\
= & \left(D u^{\zeta} \circ \phi_{\eta+\zeta}-D \tilde{u} \circ \phi_{\eta+\zeta}\right) \\
& +\left(D \tilde{u} \circ \phi_{\eta+\zeta}-D \tilde{u} \circ \phi_{\eta}-D^{2} \tilde{u} \circ \phi_{\eta} \zeta\right) \\
& +\left(D^{2} \tilde{u} \circ \phi_{\eta}-\mathcal{L}_{\eta, f}\right) \zeta+\left(D \tilde{u} \circ \phi_{\eta}-D u \circ \phi_{\eta}\right) \\
= & D\left(u^{\zeta}-\tilde{u}\right) \circ \phi_{\eta+\zeta}+D(\tilde{u}-u) \circ \phi_{\eta} \\
& +D^{2}(\tilde{u}-u) \circ \phi_{\eta} \zeta \\
& +\left(D \tilde{u} \circ \phi_{\eta+\zeta}-D \tilde{u} \circ \phi_{\eta}-D^{2} \tilde{u} \circ \phi_{\eta} \zeta\right) \\
& +\left(D^{2} u \circ \phi_{\eta}-\mathcal{L}_{\eta, f}\right) \zeta,
\end{aligned}\right.
$$

where $u^{\zeta}$ is the solution to the contact problem with contact zone described by the boundary $\Gamma^{\eta+\zeta}$ and $\tilde{u}$ represents an extension of $u$ in a neighborhood of $\Gamma^{\eta}$. Since $u$ and $\tilde{u}$ and their derivatives coincide on $\Gamma^{\eta}$ we have

$$
\begin{aligned}
\mathcal{T}(\eta+\zeta, f)-\mathcal{T}(\eta, f)-\mathcal{L}_{\eta, f} \zeta= & D\left(u^{\zeta}-\tilde{u}\right) \circ \phi_{\eta+\zeta} \\
& +\left(D \tilde{u} \circ \phi_{\eta+\zeta}-D \tilde{u} \circ \phi_{\eta}-D^{2} \tilde{u} \circ \phi_{\eta} \zeta\right) \\
& +\left(D^{2} u \circ \phi_{\eta}-\mathcal{L}_{\eta, f}\right) \zeta,
\end{aligned}
$$

and we obtain upper bounds separately for each term of the right-hand side of Eq. (5.10). 
First step. We consider the last term of (5.10). The solution $(u, \varphi)$ of problem (5.2) can easily be interpreted as the unique solution to the following bilateral Dirichlet-Neumann problem

$$
\begin{cases}-[u, \widehat{\varphi}]-[\varphi, \widehat{\theta}]=\frac{f}{2 h} & \text { in } \Omega / I^{\eta}, \\ \Delta^{2} \varphi+E[u, \widehat{\theta}]=0 & \text { in } \Omega, \\ u=0 & \text { in } I^{\eta}, \\ \left\langle\nabla u, N^{\eta}\right\rangle \circ \phi_{\eta}=\frac{1}{\left|\cos \alpha_{\eta}\right|} \mathcal{T}(\eta, f) & \text { on } \Gamma^{\eta}, \\ u=1, \quad \varphi=0, \quad \partial_{\nu} \varphi=0 & \text { on } \partial \Omega .\end{cases}
$$

This problem is well-posed with Hadamard's definition. Therefore, if we consider the associated solution $(\hat{u}, \hat{\varphi})$ of the bilateral Dirichlet-Neumann problem with homogeneous Neumann boundary condition on $\Gamma^{\eta}$

$$
\begin{cases}-[\hat{u}, \hat{\varphi}]-[\hat{\varphi}, \hat{\theta}]=\frac{f}{2 h} & \text { in } \Omega / I^{\eta}, \\ \Delta^{2} \hat{\varphi}+E[\hat{u}, \widehat{\theta}]=0 & \text { in } \Omega, \\ \hat{u}=0 & \text { in } I^{\eta}, \\ \left\langle\nabla \hat{u}, N^{\eta}\right\rangle \circ \phi_{\eta}=0 & \text { on } \Gamma^{\eta} \\ \hat{u}=1, \quad \hat{\varphi}=0, \quad \partial_{\nu} \hat{\varphi}=0 & \text { on } \partial \Omega,\end{cases}
$$

we have the bound

$$
\left\|D^{2}(\hat{u}-u) \circ \phi_{\eta}\right\|_{\sigma-1} \leq C\|\mathcal{T}(\eta, f)\|_{\sigma},
$$

where $C$ is a positive bound which depends only on the data $\left(\Omega, I^{\eta}, \frac{f}{2 h}, \widehat{\varphi}, \widehat{\theta}\right)$. Thus if we denote by $\mathcal{L}_{\eta, f}$ the second-order derivative of $\hat{u}$ along $\Gamma^{\eta}$ we obtain

$$
\left\|D^{2} u \circ \phi_{\eta}-\mathcal{L}_{\eta, f}\right\|_{\sigma-1} \leq C\|\mathcal{T}(\eta, f)\|_{\sigma}
$$

Second step. The following result proved in [21], gives a bound to the first-order derivative $D \tilde{u}$, with $\lambda=\sigma-1$ :

$$
\left\|D \tilde{u} \circ \phi_{\eta+\zeta}-D \tilde{u} \circ \phi_{\eta}-D^{2} \tilde{u} \circ \phi_{\eta} \zeta\right\|_{\sigma-1} \leq C\|D \tilde{u}\|_{\sigma+1}\|\zeta\|_{\sigma-1}^{2} .
$$

Third step. Finally let us consider the first term $(5.10)$, the pair $\left(u^{\zeta}, \varphi^{\zeta}\right)$ is solution to the problem

$$
\begin{cases}-\left[u^{\zeta}, \widehat{\varphi}\right]-\left[\varphi^{\zeta}, \widehat{\theta}\right]=\frac{f}{2 h} & \text { in } \Omega / I^{\eta+\zeta}, \\ \Delta^{2} \varphi^{\zeta}+E\left[u^{\zeta}, \widehat{\theta}\right]=0 & \text { in } \Omega, \\ \Delta^{2} \varphi^{\zeta}=0 & \text { in } I^{\eta+\zeta}, \\ u^{\zeta}=0 & \text { on } \Gamma^{\eta+\zeta}, \\ u^{\zeta}=1, \quad \varphi^{\zeta}=0, \quad \partial_{\nu} \varphi^{\zeta}=0 & \text { on } \partial \Omega .\end{cases}
$$


Therefore, $(v, \psi)=\left(u^{\zeta}-\tilde{u}, \varphi^{\zeta}-\tilde{\varphi}\right)$ is solution to

$$
\begin{cases}-[v, \widehat{\varphi}]-[\psi, \widehat{\theta}]=F=\frac{f}{2 h}-\frac{\tilde{f}}{2 h} & \text { in } \Omega / I^{\eta+\zeta}, \\ \Delta^{2} \psi+E[v, \widehat{\theta}]=0 & \text { in } \Omega, \\ \Delta^{2} \psi=0 & \text { in } I^{\eta+\zeta}, \\ v=-\tilde{u} & \text { on } \Gamma^{\eta+\zeta}, \\ v=0, \quad \psi=0, \quad \partial_{\nu} \psi=0 & \text { on } \partial \Omega,\end{cases}
$$

and we get (see Eq. (A.4) of Lemma A.2)

$$
\|v\|_{\lambda} \leq C\left[\|F\|_{\lambda-2}+\|A\|_{\lambda}+\|\eta\|_{\lambda}\left(\|F\|_{s}+\|A\|_{\sigma}\right)\right]
$$

in which we have to estimate $\|F\|_{\lambda-2}$ and $\|A\|_{\lambda}$. Let us note that $F=0$ in $\left(\Omega / I^{\eta}\right) \cap$ $\left(\Omega / I^{\eta+\zeta}\right)$ and the distance between $\Gamma^{\eta}$ and $\Gamma^{\eta+\zeta}$ is less or equal to $\|\zeta\|_{0}$ hence $\|F\|_{\sigma-2} \leq\|F\|_{\sigma}\|\zeta\|_{0} \leq C\|\zeta\|_{0}^{2}$. Next, to estimate $\tilde{u} \circ \phi_{\eta+\zeta}$ we recall that $\tilde{u}=0$, $D \tilde{u} \circ \phi_{\eta}=\mathcal{T}(\eta, f)$ on $\Gamma_{\eta}$ and once more we make use of Lemma A.1 to get:

$$
\left\{\begin{array}{l}
A=\tilde{u} \circ \phi_{\eta+\zeta}=\left(\tilde{u} \circ \phi_{\eta+\zeta}-\tilde{u} \circ \phi_{\eta}-D \tilde{u} \circ \phi_{\eta} \zeta\right)+D \tilde{u} \circ \phi_{\eta} \zeta, \\
\|A\|_{\sigma} \leq C\left(\|\tilde{u}\|_{\sigma+2}\|\zeta\|_{0}^{2}+\|\mathcal{T}(\eta, f)\|_{\sigma}\|\zeta\|_{\sigma}\right) .
\end{array}\right.
$$

\subsection{Finally}

The proof of Theorem 4.1 can now be completed using the two following results.

Proposition 5.8. Let $\eta$ and $f$ satisfying $\mathcal{T}(\eta, f)=0$, and $\tilde{u}^{\eta}$ be the restriction in $\Omega / I^{\eta}$ of the component $u$ of the solution of the bilateral problem of Lemma 5.1. Then $\tilde{u}^{\eta}$ is strictly positive in $\Omega / I^{\eta}$ for $\eta$ sufficiently small.

Proof. The proof is a simple consequence of Theorem 5.2 and Lemma 5.6.

Corollary 5.9. Let $\Omega$ be a bounded smooth domain of the plane, and $I^{\eta}$ a subdomain of $\Omega$ where $\partial I^{\eta}$ is a smooth Jordan curve with $\partial I^{\eta} \cap \partial \Omega=\emptyset$. Let problem (5.2) be given in $\Omega / I^{\eta}$ and let $\left(\tilde{u}^{\eta}, \tilde{\varphi}^{\eta}\right)$ be its solution associated with a smooth force $f$. Using the notations of Theorem 4.2, assume the component $\tilde{u}^{\eta}$ of the solution satisfies the following conditions:

(i) $\mathcal{T}(\eta, f)=0$,

(ii) $\tilde{u}^{\eta}>0$ in $\Omega / I^{\eta}$,

(iii) $\exists \delta_{0}>0$, such that $-f \geq \delta_{0}>0$.

Then the pair $\left(u^{\eta}, \varphi^{\eta}\right)$, where function $u^{\eta}$ is identically equal to $\tilde{u}^{\eta}$ in $\Omega / I^{\eta}$ and identically equal to zero in $I^{\eta}$, and function $\varphi^{\eta}$ is given by problem

$$
\begin{cases}\Delta^{2} \varphi^{\eta}+E\left[u^{\eta}, \widehat{\theta}\right]=0 & \text { in } \Omega, \\ \varphi^{\eta}=0, \quad \partial_{n} \varphi^{\eta}=0 & \text { on } \partial \Omega,\end{cases}
$$

is the solution to problem (2.4). 


\section{Concluding Remarks}

We have given a stability result for the free boundary in the case of an obstacle problem for a shallow membrane shell. Justifying a smoothness assumption by a conjecture, it was established that the solution varies smoothly as the load varies smoothly. In the case at hand the structure was linearly elastic at small strains, so that the nonlinearity arised only from the unilateral contact conditions. The case of nonlinear shells or of linearly elastic shells at large strains seems for the moment out of reach in general for at least two reasons. The first one being that the lack of uniqueness would imply a bifurcation theory for the path-following of the solution of free boundary problems, and moreover that one does not even have a general existence result for the corresponding bilateral problem. The second one is that the effect of the coupling between these two kinds of nonlinearities is not clear and has been dealt with only in the case of very simple models with one or two degrees of freedom [15].

The result given in the present work extends Schaeffer's work to the case of a membrane shell, that means to the vectorial case where the coupling between the components of the unknown vector field is due to the curvature. Although several steps are close to those given in [21], we stress the fact that the present work gives the complete proof of the results concerning differential geometry, and also gives the full explicit calculations in the boundary value problems involving the variation of the free boundary. Now extending this result to the case of a structure having a bending stiffness, does not seem to be out of reach, certainly using estimates for the biharmonic operator already taken into account in previous works [14]. The problem would be deduced from Sec. 2 of the present work and reads

$$
\begin{cases}\frac{2 E h^{3}}{3\left(1-\nu^{2}\right)} \Delta^{2} u-2 h([u, \widehat{\varphi}]+[\varphi, \widehat{\theta}])=f+\mu & \text { in } \Omega, \\ \Delta^{2} \varphi+E[u, \widehat{\theta}]=0 & \text { in } \Omega, \\ \mu \geq 0, \quad u \geq 0, \quad \mu u=0 & \text { in } \Omega, \\ u=1, \quad \partial_{n} u=0, \quad \varphi=0, \quad \partial_{n} \varphi=0 & \text { on } \partial \Omega .\end{cases}
$$

Two kinds of results remain necessary to complete the present study. The first one concerns the regularity of the solution to the free boundary problem, which has widely been studied in the scalar case and is required in the case studied here for writing the match conditions along the free boundary. The second result to be obtained is related to the fact that the whole analysis is based upon a regularity conjecture. Although there are precise counterexamples or simple numerical or physical experiments, it remains open and probably difficult. The point is the need of a connection between smoothness assumptions on the data, and the smoothness of the free boundary. 


\section{Acknowledgments}

Professor Michelle Shatzman discussed the question of the coupling in this kind of free boundary problems on the occasion of a lecture given by the first author. The present paper is dedicated to her memory.

While preparing this paper, the second author was partly supported by Fudan University at Shanghai where she stayed on the kind invitation of Professor Li Daqian.

\section{Appendix A}

\section{A.1. Proof of Lemma 3.3}

We start with the following straightforward computation: For all sufficiently smooth $(u, v, \varphi, \psi, \widehat{\theta})$ integrations by parts yield

$$
\int_{\Omega}[u, \widehat{\theta}] v d x=-a_{\widehat{\theta}}(u, v)+b_{\widehat{\theta}}(u, v)=-\int_{\Omega} \nabla u^{t} D_{2} \widehat{\theta} \nabla v d x+\int_{\partial \Omega} v \nabla u^{t} D_{2} \widehat{\theta} n d s
$$

and

$$
\begin{aligned}
\int_{\Omega}\left(\Delta^{2} \varphi\right) \psi d x & =a_{\Delta}(\varphi, \psi)+b_{\Delta}(\varphi, \psi) \\
& =\int_{\Omega} \Delta \varphi \Delta \psi d x+\int_{\partial \Omega}\left(-\Delta \varphi \partial_{\beta} \psi+\partial_{\beta}(\Delta \varphi) \psi\right) n_{\beta} d s
\end{aligned}
$$

with an obvious definition of the functionals $a_{\widehat{\theta}}, b_{\widehat{\theta}}, a_{\Delta}, b_{\Delta}$.

The bilinear form $a_{\widehat{\theta}}$ is coercive since $D_{2} \widehat{\theta}$ is assumed to be positive definite and we get

$$
a_{\widehat{\theta}}(u, \varphi) \leq C_{\widehat{\theta}}\|\nabla u\|\|\nabla \varphi\|,
$$

where $C_{\widehat{\theta}}$ is the continuity constant. We are now in a position to prove Lemma 3.3. We note that $(v, \psi) \in K(\Omega)$ implies $b_{\widehat{\theta}}(\varphi, v)=0, b_{\Delta}(\varphi, \psi)=0, b_{\widehat{\theta}}(u, \psi)=0$ where $K(\Omega)$ has been defined as

$$
K(\Omega)=\left\{(v, \psi) \in H^{1}(\Omega) \times H_{0}^{2}(\Omega), v \geq 0 \text { in } \Omega, v=1 \text { on } \partial \Omega\right\} .
$$

Then

$$
\left\{\begin{array}{l}
\int_{\Omega}(-[u, \widehat{\varphi}]-[\varphi, \widehat{\theta}]) v d x=a_{\widehat{\varphi}}(u, v)+a_{\widehat{\theta}}(\varphi, v)-b_{\widehat{\varphi}}(u, v)-b_{\widehat{\theta}}(\varphi, v), \\
\int_{\Omega}\left(\Delta^{2} \varphi+E[u, \widehat{\theta}]\right) \psi d x=a_{\Delta}(\varphi, \psi)+b_{\Delta}(\varphi, \psi)-E a_{\widehat{\theta}}(u, \psi)+E b_{\widehat{\theta}}(u, \psi) .
\end{array}\right.
$$

We multiply the first equation by a positive parameter $\beta$ and add the second one, then we define the following bilinear form,

$$
A((u, \varphi),(v, \psi))=\beta a_{\widehat{\varphi}}(u, v)+\beta a_{\widehat{\theta}}(\varphi, v)+a_{\Delta}(\varphi, \psi)-E a_{\widehat{\theta}}(u, \psi) .
$$


Due to Poincaré inequality $\|\Delta \varphi\| \geq C_{P}\|\nabla \varphi\|$ we have

$$
\left\{\begin{aligned}
A((u, \varphi),(u, \varphi)) & \\
& =\beta a_{\widehat{\varphi}}(u, u)+\beta a_{\widehat{\theta}}(u, \varphi)+a_{\Delta}(\varphi, \psi)-E a_{\widehat{\theta}}(u, \varphi) \\
& =\beta a_{\widehat{\varphi}}(u, u)+a_{\Delta}(\varphi, \varphi)+(-E+\beta) a_{\widehat{\theta}}(u, \varphi) \\
& \geq \beta C_{\widehat{\varphi}}\|\nabla u\|^{2}+\|\Delta \varphi\|^{2}-|-E+\beta| C_{\widehat{\theta}}\|\nabla u\|\|\nabla \varphi\| \\
& \geq\left(\beta C_{\widehat{\varphi}}-\frac{|-E+\beta| C_{\widehat{\theta}} \alpha}{2}\right)\|\nabla u\|^{2}+\left(C_{P}^{2}-\frac{|-E+\beta| C_{\widehat{\theta}}}{2 \alpha}\right)\|\nabla \varphi\|^{2},
\end{aligned}\right.
$$

where $\alpha$ is a positive parameter to be adjusted together with $\beta>0$ in order to obtain the coercivity of $A$

$$
\beta C_{\widehat{\varphi}}-\frac{|-E+\beta| C_{\widehat{\theta}} \alpha}{2}>0 \text { and } C_{P}^{2}-\frac{|-E+\beta| C_{\widehat{\theta}}}{2 \alpha}>0 .
$$

Let us observe that it is always possible to find $\beta$ such that

$$
\frac{|-E+\beta| C_{\widehat{\theta}}}{2 C_{P}^{2}}<\frac{2 \beta C_{\widehat{\varphi}}}{|-E+\beta| C_{\widehat{\theta}}} \quad \text { or } \quad|-E+\beta|^{2}<k \beta \quad \text { with } k>0 \text { large enough. }
$$

This implies that Eq. (A.2) amounts to finding $\alpha$ such that

$$
0<\frac{|-E+\beta| C_{\widehat{\theta}}}{2 C_{P}^{2}}<\alpha<\frac{2 \beta C_{\widehat{\varphi}}}{|-E+\beta| C_{\widehat{\theta}}},
$$

which is obviously always possible. The bilinear form $A$ is continuous and coercive due to the positivity of $D_{2} \widehat{\varphi}$. Thus existence and uniqueness of the solution of (3.3) follow as a direct application of variational inequalities theory [16].

\section{A.2. Some Hölder estimates}

In this appendix, we recall without proof two results on Hölder norms given in [21] which allow to cope with our problem.

Lemma A.1. Let $\Gamma^{0}$ and $\phi_{\eta}$ be defined as in Eq. (5.1), and let $0<s<1$ and $1 \leq \lambda \leq \sigma+2=s+4$.

(i) For any function $g$ on $\mathbb{R}^{n}$ there exists a strictly positive constant $C$ such that [21, Eq. (3.3)]:

$$
\left\|g \circ \phi_{\eta}\right\|_{\lambda} \leq C\left(\|g\|_{\lambda}+\|g\|_{1}\|\eta\|_{\lambda}\right) \text {. }
$$

(ii) Let $w$ be a function on $\mathbb{R}^{n}$ defined on a neighborhood of $\Gamma^{0}$. There exists a strictly positive constant $C$ such that for any $\eta \in C^{k}\left(\Gamma^{0}\right), \eta+\zeta \in C^{k}\left(\Gamma^{0}\right)$ [21, Lemma 4.2] we have:

$$
\left\|w \circ \phi_{\eta+\zeta}-w \circ \phi_{\eta}-\zeta D w \circ \phi_{\eta}\right\|_{\lambda} \leq C\|w\|_{\lambda+2}\|\zeta\|_{\lambda}^{2}
$$


Lemma A.2. We consider the following Dirichlet coupled problem for unknowns $(v, \psi)$ with data $(F, A)$ :

$$
\begin{cases}-[v, \widehat{\varphi}]-[\psi, \widehat{\theta}]=F & \text { in } \Omega / I^{\eta}, \\ \Delta^{2} \psi+E[v, \widehat{\theta}]=0 & \text { in } \Omega, \\ \Delta^{2} \psi=0 & \text { in } I^{\eta}, \\ v=A & \text { on } \Gamma^{\eta}, \\ v=1, \quad \psi=0, \quad \partial_{\nu} \psi=0 & \text { on } \partial \Omega .\end{cases}
$$

For $\lambda$ noninteger, $\lambda>2$, and $\|\eta\|_{\sigma} \leq \varepsilon$ there exists a strictly positive constant $C$ such that we have:

$$
\|v\|_{\lambda} \leq C\left[\|F\|_{\lambda-2}+\|A\|_{\lambda}+\|\eta\|_{\lambda}\left(\|F\|_{s}+\|A\|_{\sigma}\right)\right] .
$$

\section{References}

[1] S. Alinhac and P. Gérard, Opérateurs Pseudo-différentiels et Théorème de NashMoser (InterEditions et CNRS Editions, Paris, 1991).

[2] A. Bonnet and R. Monneau, Distribution of vortices in a type II superconductor as a free boundary problem: Existence and regularity via Nash-Moser theory, Interfaces Free Bound 2(2) (2000) 181-200.

[3] L. Caffarelli and A. Friedman, The obstacle problem for the biharmonic operator, Ann. Scuola Norm. Sup. Pisa 6(4) (1979) 151-184.

[4] L. Cherfils, A. Léger and B. Miara, On the regularity of the solution in a free boundary problem for a shallow shell, in progress.

[5] P.-G. Ciarlet and B. Miara, A justification of the two-dimensional equations of a linearly elastic shallow shell, Comm. Pure Appl. Math. 45 (1993) 327-360.

[6] P.-G. Ciarlet and J.-C. Paumier, A justification of the Marguerre-von Kármán shell equations, Comput. Mech. 1 (1986) 177-202.

[7] A. Cimetière and A. Léger, Un résultat de différentiabilité dans le problème d'obstacle pour des poutres en flexion, C. R. Acad. Sci. I 316 (1994) 749-754.

[8] R. Hamilton, The inverse function theorem of Nash and Moser, Bull. Amer. Math. Soc. 7(1) (1982) 65-222.

[9] D. Kinderleherer and L. Nirenberg, Regularity in free boundary problems, Ann. Scuola Norm. Super. Pisa Cl. Sci. (4) 4(2) (1977) 373-391.

[10] D. Kinderleherer and G. Stampacchia, An Introduction to Variational Inequalities and Their Applications (Academic Press, New York, 1980).

[11] A. Léger and B. Miara, Mathematical justification of the obstacle problem in the case of a shallow shell, J. Elasticity 90 (2008) 241-257.

[12] A. Léger and B. Miara, The obstacle problem for shallow shells: A curvilinear approach, Int. J. Numer. Anal. Model. Ser. B 2(1) (2011) 1-26.

[13] A. Léger and C. Pozzolini, Sur la zone de contact entre une plaque élastique et un obstacle rigide, C. R. Mécanique 355 (2007) 144-149.

[14] A. Léger and C. Pozzolini, A stability result concerning the obstacle problem for a plate, J. Math. Pures Appl. 90(6) (2008) 505-519.

[15] A. Léger, E. Pratt and Q. J. Cao, A fully nonlinear oscillator with contact and friction, Nonlinear Dynam. 70 (2012) 511-522.

[16] J.-L. Lions and G. Stampacchia, Variational inequalities, Comm. Pure Appl. Math. 22(2) (1969) 153-188. 
[17] R. Monneau, On the number of singularities for the obstacle problem in two dimensions, J. Geom. Anal. 13(2) (2003) 359-389.

[18] E. Sanchez-Palencia and J. Sanchez-Hubert, Coques Élastiques Minces, Propriétés Asymptotiques (Masson, Paris, 1997).

[19] D. G. Schaeffer, An example of generic regularity for a nonlinear elliptic equation, Arch. Ration. Mech. Anal. 57 (1974) 134-141.

[20] D. G. Schaeffer, The capacitor problem, Indiana Univ. Math. J. 24(12) (1975) 11431167.

[21] D. G. Schaeffer, A stability theorem for the obstacle problem, Adv. Math. 16 (1975) $34-47$.

[22] D. G. Schaeffer, Some example of singularities in a free boundary, Ann. Scuola Norm. Sup. Pisa Cl. Sci. (4) 4(1) (1977) 133-144.

[23] R. Schumann, Regularity for Signorini's problem in linear elasticity, Manuscripta Math. 63 (1989) 255-291.

[24] J. T. Schwartz, Nonlinear Functional Analysis (Courant Institute of Mathematical Sciences, New York University, 1965). 